\title{
Circadian Clock in Cell Culture: II. In vitro Photic Entrainment of Melatonin Uscillation from Dissociated Chick Pineal Cells
}

\author{
Linda M. Robertson and Joseph S. Takahashi \\ Department of Neurobiology and Physiology, Northwestern University, Evanston, Illinois 60201
}

The avian pineal gland contains circadian oscillators that regulate the rhythmic synthesis of melatonin. We have developed a flow-through cell culture system in order to begin to study the cellular and molecular basis of this vertebrate circadian oscillator. Pineal cell cultures express a circadian oscillation of melatonin release for at least 5 cycles in constant darkness with a period close to $24 \mathrm{hr}$. In all circadian systems, light regulates the rhythm by the process of entrainment that involves control of the phase and period of the circadian oscillator. In chick pineal cell cultures we have investigated the entraining effects of light in 2 ways: by shifting the light-dark cycle in vitro and by measuring the phaseshifting effects of single light pulses. A 6 hr advance or delay of a LD 12:12 light-dark cycle produced a corresponding shift in the melatonin rhythm. The phase shifts of the rhythms persisted after transfer to constant darkness, showing that the underlying circadian oscillator was entrained. Photic entrainment of the oscillator was further characterized by measuring the phase-shifting effects of single $6 \mathrm{hr}$ light pulses. Single pulses of light shifted the phase of the circadian oscillator in a phase-dependent manner. Light pulses beginning early in the subjective night delayed the phase of the oscillation $8 \mathrm{hr}$ relative to dark controls. Conversely, light pulses beginning late in the subjective night advanced the phase of the oscillation nearly $8 \mathrm{hr}$. Thus, photoreceptors within the cell cultures can mediate entrainment of the pineal oscillators. Phase-dependent phase shifts to light pulses (as described in a "phase-response curve") are a fundamental characteristic of all circadian systems. The expression of this circadian property in the pineal cell cultures should permit a mechanistic analysis of the photic entrainment pathway.

Environmental light cycles regulate or entrain circadian rhythms by shifting the phase of the circadian oscillator underlying the rhythm. Although the formal properties of entrainment have been thoroughly studied (Pittendrigh, 1981), little information exists concerning the cellular and biochemical mechanisms that regulate circadian oscillators, especially among vertebrates (Takahashi and Zatz, 1982; Jacket, 1984; Takahashi and Menaker, 1984a). In birds and reptiles, the isolated pineal gland contains circadian oscillators, with photoreceptive input, which

\footnotetext{
Received Dec. 31, 1986; revised July 16, 1987; accepted July 16, 1987.

This work was supported by NIMH Grant MH-39552, NSF Presidential Young Investigator Award DCB-8451642, and Searle Scholars Award 85-H-107 to J.S.T.

Correspondence should be addressed to Dr. Takahashi, Department of Neurobiology and Physiology, Northwestern University, Hogan Hall, Evanston, IL 60201 .

Copyright (C) 1988 Society for Neuroscience $0270-6474 / 88 / 010022-09 \$ 02.00 / 0$
}

regulate the rhythmic synthesis of melatonin (Takahashi et al., 1980; Menaker and Wisner, 1983; Takahashi and Menaker, 1984b). Previous work has shown that light exposure in vitro can modulate $N$-acetyltransferase activity and melatonin production in chick pineal organ cultures (Deguchi, 1979a, 1981; Wainwright and Wainwright, 1980; Hamm et al., 1983; Takahashi and Menaker, 1984b). Although acute exposure to light can suppress melatonin synthesis, photic entrainment of circadian rhythms in the pineal in vitro has not been definitively demonstrated. Preliminary work has suggested that entrainment may occur; however, none of these studies demonstrated that the steady-state phase of the oscillator was regulated by light (Deguchi, 1979b; Kasal and Perez-Polo, 1980; Takahashi and Menaker, 1984b).

In the previous paper we reported that dissociated chick pineal cell cultures express a circadian oscillation of melatonin release (Robertson and Takahashi, 1988). The rhythm persists in constant darkness for at least 5 cycles; however, the amplitude of the rhythm damps. When maintained in a light-dark cycle the cells express a high-amplitude mclatonin rhythm. The maintenance of a high-amplitude oscillation in a light-dark cycle indirectly suggests that the dispersed pineal cells are photoreceptive in culture. In addition, the rhythm does not appear to be entirely driven by the light-dark cycle, but rather exhibits an anticipatory increase prior to lights out and a decrease prior to lights on. Furthermore, constant light exposure lengthens the free-running period of the melatonin rhythm. The effects of light on the period length demonstrate that photic information has input to the circadian oscillator. Taken together, these results suggest that light may be capable of entraining the circadian oscillation in pineal cell cultures in vitro. We have tested this hypothesis directly by investigating the effects of shifted lightdark cycles and the effects of single light pulses upon the steadystate phase of the melatonin rhythm in pineal cell cultures. We report here that light exposure in vitro can entrain the circadian oscillation of melatonin release from dissociated chick pineal cells.

\section{Materials and Methods}

Animals

Newly hatched male chicks (Gallus domesticus, white leghorn) were purchased from Cornbelt hatcheries (Forest, IL). The animals were raised under $12 \mathrm{hr}$ light: $12 \mathrm{hr}$ dark (LD 12:12) lighting regime with lights on at 0700 CST for 3-6 weeks prior to study. Food (Purina Chick Startena) and water were available continuously.

\section{Cell culture}

The flow-through cell culture procedure used in these experiments has been described in detail in the preceding paper (Robertson and Takahashi, 1988). For each experiment 10 pineal glands were enzymatically 


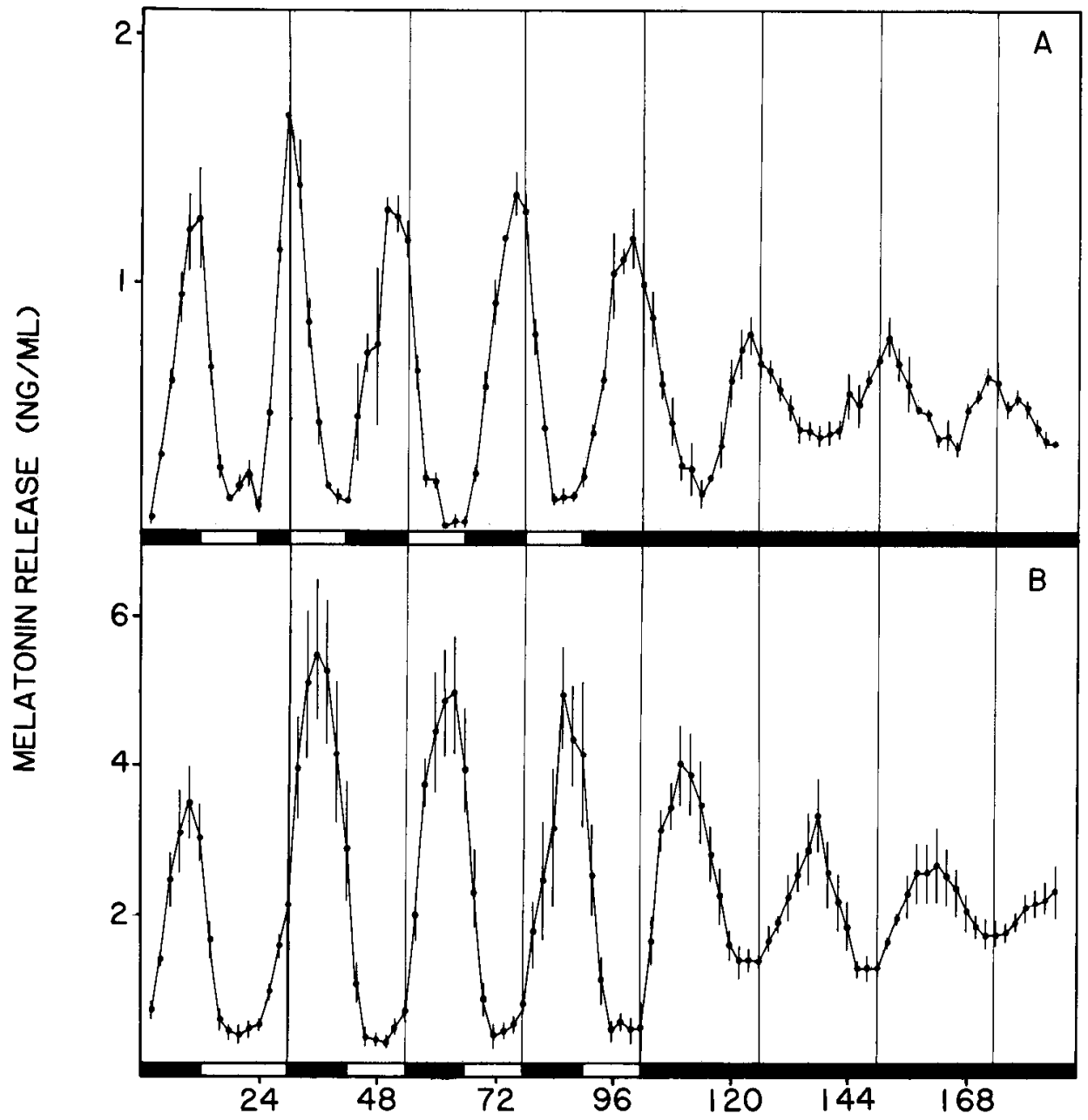

TIME IN HOURS
Figure 1. In vitro entrainment of circadian oscillation of melatonin release from dispersed chick pineal cells. Points and bars represent mean \pm SEM of 3 replicate cell chambers. Dark bars indicate the light-dark cycle. Flow rate was $0.5 \mathrm{ml} / \mathrm{hr}$. All cell chambers were exposed to LD 12:12 to ensure initial synchrony of the oscillation. $A$, Six-hour advance of light-dark cycle advanced the melatonin oscillation in cyclic light and constant conditions. $B$, Six-hour delay of light-dark cycle delayed the melatonin oscillation in cyclic light and constant conditions. dispersed with collagenase $(1 \mathrm{mg} / \mathrm{ml})$ and collected by differential centrifugation. The dispersed cells were maintained in culture medium with the following composition: Medium-199 with Hank's salts and L-glutamine (GIBCO, \#400-1200) supplemented with 10 mM HEPES buffer (US Biochem), $5 \%$ fetal bovine serum (Biologos, $\# 1210378$ ), 10\% heatinactivated horse serum (GIBCO, \#200-2050), $100 \mathrm{U} / \mathrm{ml}$ penicillin $\mathrm{G}$, $100 \mu \mathrm{g} / \mathrm{ml}$ streptomycin, and $0.9 \mathrm{mg} / \mathrm{ml} \mathrm{NaHCO}_{3}$ supplemented for $5 \%$ $\mathrm{CO}_{2}$ atmosphere. Cytodex 3, collagen-coated microcarriers (Pharmacia) were used as a substrate for cell attachment. The cells were maintained in an incubator at $37^{\circ} \mathrm{C}$ with $95 \%$ air $/ 5 \% \mathrm{CO}_{2}$ and exposed to $\mathrm{LD} 12$ : 12 lighting conditions. Half the medium was changed every other day. After 4-5 d in culture, the cells were loaded into the flow-through apparatus described in the previous paper. The cell chambers were houscd within light-controlled boxes fitted with a $4 \mathrm{~W}$ fluorescent bulb and a Schott KG1 heat filter. The entire apparatus was maintained at $37^{\circ} \mathrm{C}$. Approximately $1-2 \times 10^{5}$ cells were loaded into each chamber. For flow-through experiments an air-equilibrated medium was used with the following composition: Medium-199 with Hank's salts and L- glutamine supplemented with $10 \mathrm{mM}$ HEPES buffer, $5 \%$ fetal bovine serum, $10 \%$ heat-inactivated horse serum, and $50 \mu \mathrm{g} / \mathrm{ml}$ gentamicin. Medium was infused through the cell chambers at a rate of $0.25-0.5 \mathrm{ml} / \mathrm{hr}$ depending on the experiment. Two-hour fractions were collected and assayed for melatonin content using a specific radioimmunoassay as previously described (Takahashi et al., 1980; Robertson and Takahashi, 1988).

\section{Data analysis}

Phase reference point. The phase of the melatonin oscillation was analyzed using the midpoint of the cycle as the phase reference point. The method for calculating this reference point has been described in detail (Robertson and Takahashi, 1988). The midpoint phase reference is defined as the time midway bewteen the half-rise and half-fall of each melatonin peak.

Design of perturbation experiments. Measurement of the phase-response curve required 3 sets of experiments. One experiment involved 1 experimental group and 1 control group (4 replicates each per condition, a total of 8 cell chambers). The remaining 2 experiments involved 4 or 5 experimental groups and 1 control group (4 or 5 replicates per group, in each experiment). Each group of experimental chambers was exposed to a single pulse of light. The light intensity at the level of the chambers was $100 \mu \mathrm{W} / \mathrm{cm}^{2}$. The subsequent rhythm was assayed in constant darkness to determine the phasc of the oscillation.

Calculations of phase shifts. The phases of each culture within a given experimental group were averaged for each cycle. The mean of the experimental group was subtracted from that of a control group to determine the magnitude of the phase difference. Significance was determined using analysis of variance followed by Student's $t$ test for pairwise comparison of treatment means, and $95 \%$ confidence limits were calculated using the SDs of the 2 groups to calculate a weighted SD for the difference of the mean values.

Normalization. In order to reduce the differences among cell chambers in the absolute amplitude of the melatonin rhythm, some of the data are presented in normalized form relative to the mean value of the record. This was performed by dividing the melatonin values from each channel by the mean value of the time series of that channel and multiplying by 100 . Thus, the mean value for the time scrics from each channel corresponds to $100 \%$. This calculation eliminates variations in the amplitude of the rhythm due to procedures which change the absolute amount of melatonin. The primary source of this variation ap- 


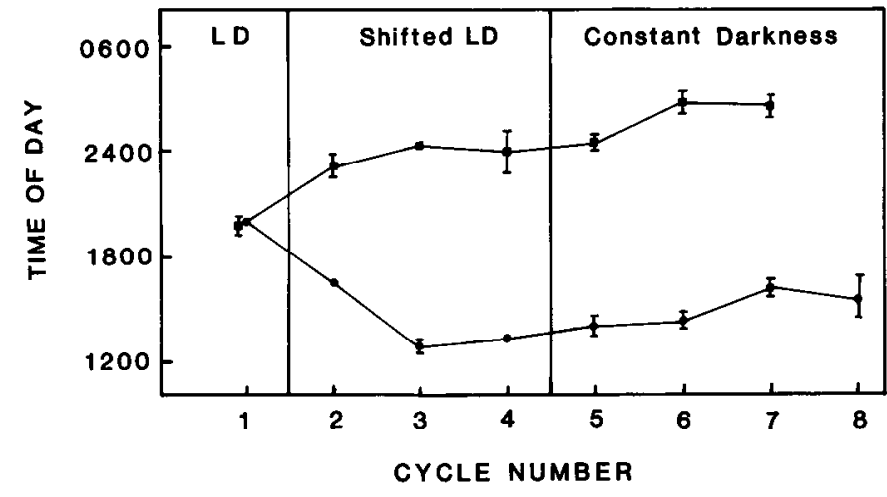

Figure 2. Analysis of the phase of the rhythms during in vitro entrainment of melatonin oscillation from dispersed chick pineal cells. The midpoint of the cycle was used as a phase reference point. Solid circles and bars represent the mean \pm SEM of 4 replicate cell chambers in which the light-dark cycle was advanced $6 \mathrm{hr}$. Solid squares and bars represent the mean \pm SEM of 4 replicate cell chambers in which the light-dark cycle was delayed $6 \mathrm{hr}$.

pears to be the number of cells loaded into each chamber. Because the phase and period of the circadian rhythm are the primary measurements of concern and because these measurements are independent of the absolute level, the normalization procedure simply enhances the visualization of phase and period when comparing records.

\section{Results}

In vitro entrainment of circadian rhythms in pineal cell cultures

In order to determine whether the melatonin rhythm from pineal cell cultures can be entrained to light, the experiment illustrated in Figure 1 was performed. Two groups of cell cultures were exposed to LD 12:12 for $24 \mathrm{hr}$. One of the groups was then exposed to 3 cycles of LD 12:12, which were advanced 6 hr (Fig. 1A). After 2 transient cycles, the shift in the light-dark cycle produced a corresponding advance in the melatonin rhythm that persisted after release into constant darkness. The second group was exposed to 3 cycles of LD 12:12 which were delayed $6 \mathrm{hr}$ (Fig. $1 B$ ). The delay in the light-dark cycle produced a delay in the melatonin rhythm that persisted after release into constant darkness. The maintenance of an advance or delay in the phase of the melatonin oscillation in constant conditions strongly suggests that a steady-state shift in the phase of the oscillation had occurred.

These observations were quantified by determining the phases of the cultures in the 2 treatments. As illustrated in Figure 2, the phases of the 2 groups of cultures were the same on the first day of the experiment. During the shifted light-dark cycle, the mean phase of the chambers exposed to the advanced light cycle occurred earlier each cycle, while the phase of the group exposed to the delayed light cycle occurred later. The phase difference between the 2 groups reached a maximum of over $11 \mathrm{hr}$ after 2 transient cycles. This phase difference was maintained in constant darkness, demonstrating that the steady-state phase of the rhythm was shifted. The slight positive slope observed during constant conditions is due to an endogenous period of greater than $24 \mathrm{hr}$. These results demonstrate that the circadian oscillators regulating the melatonin rhythm in dispersed pineal cell cultures can be entrained by light cycles in vitro.

\section{Effects of discrete light pulses}

Phase-shifting effect of light. In all circadian systems, entrainment occurs by the differential phase-shifting effects of discrete
Table 1. Determination of phase shift magnitude using the midpoint of the cycle as the phase reference point

\begin{tabular}{lrccll} 
& \multicolumn{2}{l}{\begin{tabular}{l} 
Phase reference point $(\mathrm{hr})$ \\
\cline { 2 - 3 } Cycle
\end{tabular}} & $\begin{array}{l}\text { Control } \\
(X \pm \mathrm{SD})\end{array}$ & $\begin{array}{l}\text { Experimental } \\
(X \pm \mathrm{SD})\end{array}$ & $\begin{array}{l}\text { Phase shift } \\
(\mathrm{hr})\end{array}$ \\
\hline 0 & $6.7 \pm 0.46$ & $6.7 \pm 0.73$ & $0.0 \pm 1.1$ & $\mathrm{~ns}$ \\
$1^{a}$ & $31.7 \pm 0.72$ & - & - & - \\
2 & $54.9 \pm 1.58$ & $49.8 \pm 0.52$ & $+5.1 \pm 2.03$ & $<0.001$ \\
3 & $79.6 \pm 0.85$ & $73.6 \pm 0.51$ & $+6.0 \pm 1.20$ & $<0.001$ \\
4 & $103.9 \pm 0.45$ & $98.0 \pm 1.25$ & $+5.9 \pm 2.89$ & $<0.01$ \\
5 & $127.6 \pm 1.97$ & $121.8 \pm 0.67$ & $+5.8 \pm 3.35$ & $<0.01$
\end{tabular}

a Light perturbation occurred during this cycle, obscuring the phase reference for the experimental chambers.

${ }^{b}$ Significance for each cycle was determined using Student's $t$ test.

light pulses (Pittendrigh, 1981). In order to test whether this process occurs in dispersed pineal cell cultures, we examined the phase-shifting effects of single $6 \mathrm{hr}$ light pulses on the melatonin rhythm. Figure 3 illustrates the phase-shifting effects of a $6 \mathrm{hr}$ light pulse beginning at circadian time 20 (CT 20), where CT 12 is defined as the light-to-dark transition. Two groups of cultures were used to assay the phase shift: a control group maintained in constant darkness to determine the steady-state phase of the oscillation and an experimental group exposed to the light pulse to determine the phase shift relative to the phase of the dark control. All cell chambers were exposed to LD 12: 12 for $24 \mathrm{hr}$ to ensure synchrony of the cultures. Figure $3 A$ illustrates the individual records from 4 chambers in the experimental group exposed to a $6 \mathrm{hr}$ light pulse at CT 20. Figure $3 B$ illustrates the individual records of 4 chambers in the control group in constant darkness. Figure $3 C$ shows the ensemble averages of the 2 groups plotted together to facilitate visual comparison of the phases of the rhythms. As illustrated in Figure $3 C$, the light pulse at CT 20 produced an advance shift in the phase of the circadian oscillation of melatonin release. The procedure for calculating the magnitude of phase shift is illustrated in Table 1. The light pulse at CT 20 produced an advance of approximately $6 \mathrm{hr}$ relative to unpulsed controls (Table 1 ). The shift was detectable within 1 cycle without apparent transients. Furthermore, the phase shift appears to be in steady state because the magnitude of the shift is stable for 4 cycles.

To explore the phase-shifting effect of $6 \mathrm{hr}$ light pulses at different phases of the cycle, the experiment diagrammed in Figure 4 was performed. Six-hour light pulses were given at 3 hr intervals to 8 experimental groups. The phase-shifting effects of single light pulses were dependent upon the phase of the stimulus. The ensemble averages of the 8 experimental groups and their respective control groups are shown in Figure 5. Pulses beginning in the late subjective day and early subjective night (CT 6, 9, 12, 15) produced delays of the melatonin oscillation (Fig. 5, $A, F, G, H$ ). Pulses beginning in the late subjective night (CT 18, 21, 24) produced phase advances of the rhythm (Fig. $5, B-D$ ). A light pulse beginning in the early subjective day (CT 3) had no significant effect on the phase of the circadian oscillation (Fig. $5 E$ ). Figure 6 shows the phase-response curve, which is the dependence of the direction and magnitude of the phase shift upon the phase of the light pulse, using values derived from the second cycle after the light pulse. Maximum advance phase shifts of $8 \mathrm{hr}$ were obtained with light pulses beginning at CT 


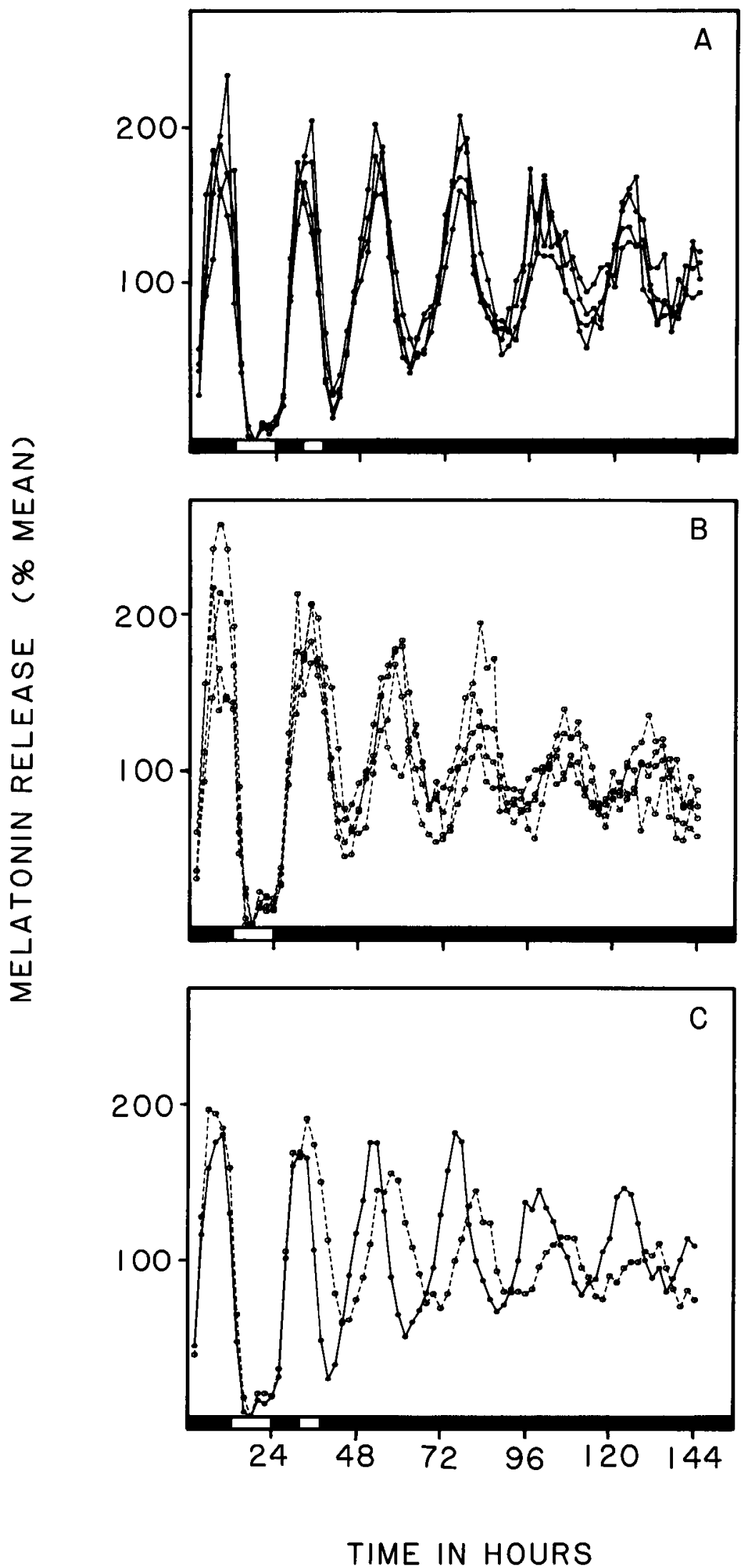

Figure 3. Light-induced phase shift of the circadian oscillation of melatonin release from dispersed chick pineal cells. Dark bars indicate the light-dark cycle. All cell chambers were exposed to LD $12: 12$ to cnsurc initial synchrony of the oscillation. Flow rate was $0.25 \mathrm{ml} / \mathrm{hr}$. $A$, Superimposed individual records from 4 replicate cell chambers that received a $6 \mathrm{hr}$ pulse of light beginning at circadian time (CT) 20. The light-todark transition is defined as CT 12 by convention. $B$, Superimposed individual records from 4 replicate cell chambers maintained in constant darkness. $C$, Superimposed ensemble averages from records presented in $A$ and $B$ illustrate light-induced phase advance of the melatonin oscillation during subsequent cycles in constant darkness. Closed circles represent mean of 4 cell chambers that received a $6 \mathrm{hr}$ light pulse and open circles represent mean of 4 cell chambers maintained in constant darkness.
18. Maximum delay phase shifts of approximately the same magnitude were obtained with light pulses beginning at CT 12 .

The time course of the phase shifts for $6 \mathrm{hr}$ light pulses is illustrated in Figure 7, which plots the magnitude of the phase shift for 3 cycles after the pulse. Advance phase shifts resulting from pulses beginning at CT 18, 21, and 24 occurred within 1 cycle. The phase shifts were stable for at least 3 cycles in constant conditions, indicating that a steady-state phase shift had been 
Figure 4. Design of light pulse perturbation experiments. Dark bars indicate the light-dark cycle. The top of figure shows a smoothed (using a Blackman filter, cutoff frequency, 0.25 cycles/ hr) ensemble average of melatonin release from cell chambers maintained in constant darkness. All cell chambers were exposed to LD 12:12 for $24 \mathrm{hr}$ to ensure initial synchrony of the oscillations. Six-hour light pulses were then initiated at sequential times (CT 15, 18, $21,24,3,6,9$, and 12 ) relative to the control oscillation.

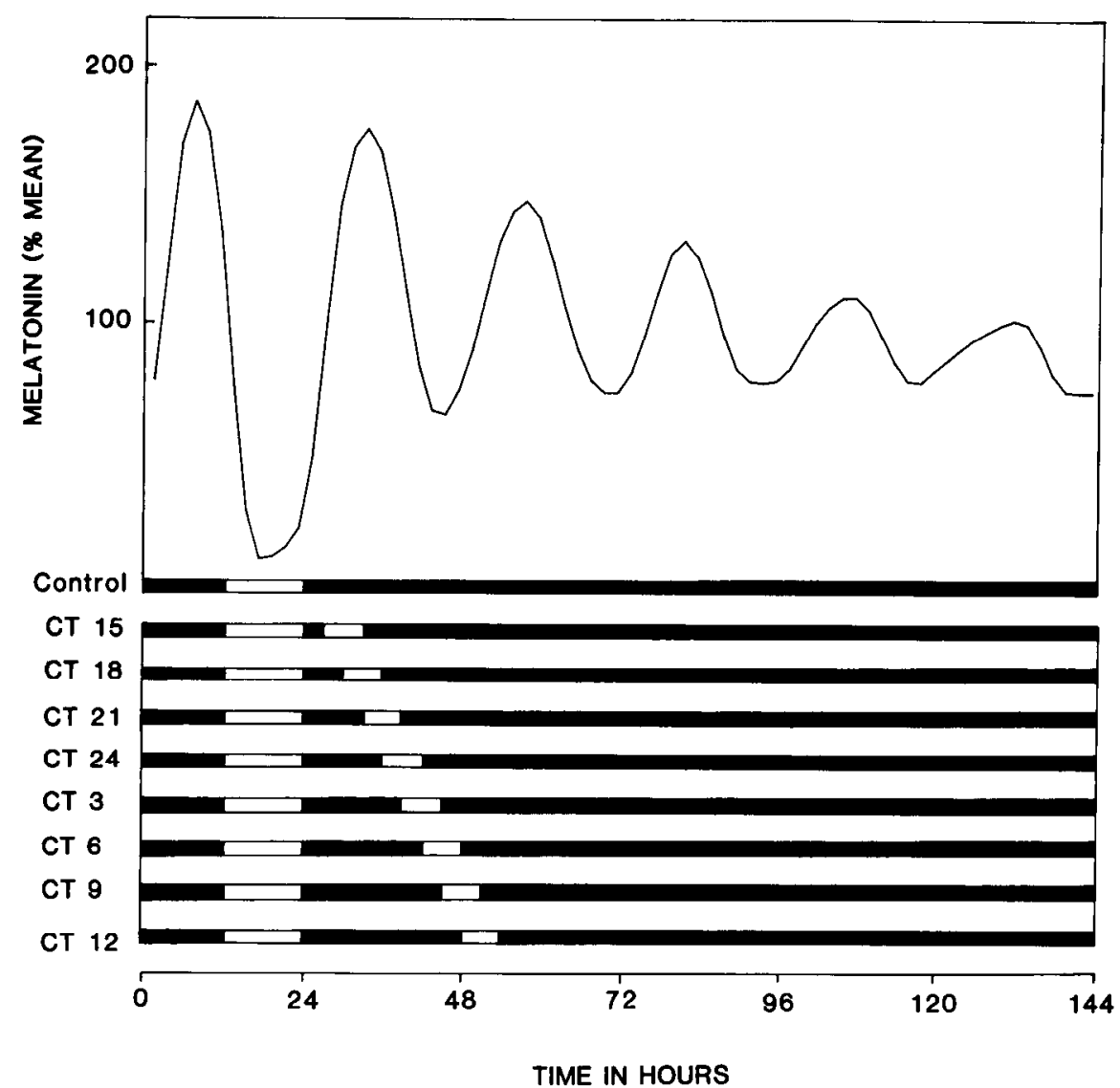

achieved. Delay phase shifts resulting from pulses beginning at CT $6,9,12$, and 15 also occurred within 1 cycle. However, for pulses at CT 6,9 , and 12 , the maximum phase shift did not occur until the second cycle following the light pulse.

To explore the effect of the duration of the light pulse on phase shifts, different duration pulses were presented at the same phase. The ensemble averages of the melatonin rhythms for this experiment are shown in Figure 8. Light pulses with different durations $(1,2,3$, and $6 \mathrm{hr})$ beginning at CT 20 all produced significant phase shifts. Figure 9 shows that the magnitude of the shift was dependent upon duration. The magnitude of the phase shift appears to increase in duration with 1,2 , and $3 \mathrm{hr}$ pulses, and there was no further increase with $6 \mathrm{hr}$.

Effects of light pulses on the amplitude of the oscillation. The circadian oscillation of melatonin release from dispersed chick pineal cells exhibited a gradual decrease in amplitude during constant darkness. This damping of the oscillation was consistent in control groups held in constant darkness; however, in experimental groups, exposure to light pulses appeared to modify the amplitude of the oscillation. In almost every case, light increased the amplitude of the subsequent cycle in constant darkness relative to controls (Fig. $5, C-H$ ). Thus, in addition to a phase-shifting effect, there was an apparent light-induced resetting of the amplitude of the oscillation. The effect on amplitude varied with the phase of the light pulse in a rather complex manner. At CT 15, there was no effect on the amplitude of the rhythm (Fig. 5A). At CT 18, the amplitude increased on the second and third cycles after the pulse, but there was little change in amplitude on the first cycle (Fig. 5B). At all other phases, the amplitude increased after the pulse and appeared to be "reset" to a value similar to the first cycle in constant darkness. In addition, the effect on amplitude was dependent on the duration of the light pulse (Fig. 8). A $6 \mathrm{hr}$ light pulse produced an increase in the amplitude of the rhythm in constant darkness. The amplitude resetting effect was diminished as the pulse duration was decreased to $3 \mathrm{hr}$ (Fig. 8B) and $2 \mathrm{hr}$ (Fig. 8C). A $1 \mathrm{hr}$ light pulse produced no detectable increase in amplitude (Fig. $8 D$ ).

There are at least 2 possible explanations for the effects of light pulses on the amplitude of the rhythm: increased coherence of a population of oscillators or amplitude resetting of a damped oscillator. The first hypothesis is an extension of the population desynchronization model described in the previous paper (Robertson and Takahashi, 1988), which postulates that the damping in amplitude of the rhythm is due to desynchronization of oscillators. If there were variability in the endogenous periods of individual oscillators in the cultures, then in constant conditions the individual oscillators would desynchronize and result in apparent damping of the overt rhythm. A single pulse of light could resynchronize the oscillators in the population and reset the amplitude of the overt rhythm (Takahashi and Menaker, 1984b). Alternatively, if the component oscillators in the pineal cultures are damped oscillators, light pulses could reset the amplitude as described by Enright (1984).

Acute inhibition of melatonin release. In addition to the effects of light upon the phase and amplitude of the oscillation, acute exposure to light causes a reduction in melatonin release. As illustrated in Figure $3 C$, melatonin release decreased dramatically during the light pulse. When light pulses were applied at different phases of the rhythm as shown in Figure 5, light exposure always caused a reduction in the melatonin release relative to the dark control group. The magnitude of the reduction in melatonin release varied with the phase of the light pulse. At 

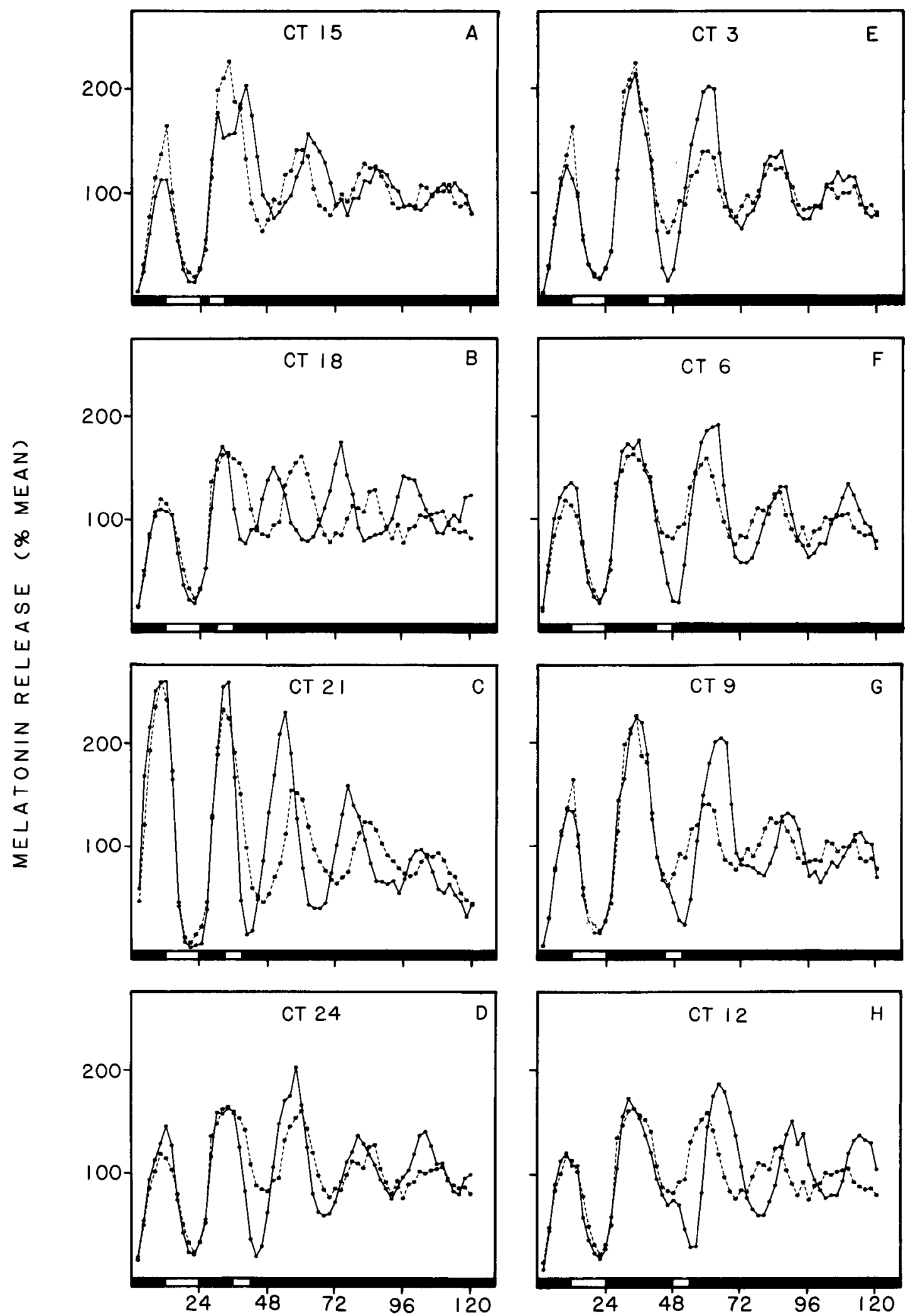

TIME IN HOURS

Figure 5. Phase-dependent effects of $6 \mathrm{hr}$ light pulses. Dark bars indicate the light-dark cycle. Closed circles represent the ensemble average of 4-5 replicate cell chambers (depending on the experiment) that received a $6 \mathrm{hr}$ pulse of light. Open circles represent the ensemble average of 4-5 replicate cell chambers (depending on the experiment) maintained in constant darkness. Flow rate was $0.25 \mathrm{ml} / \mathrm{hr}$ in all cases except CT 21 in which the flow rate was $0.5 \mathrm{ml} / \mathrm{hr}$. Light pulses were initiated at $3 \mathrm{hr}$ intervals during the first $30 \mathrm{hr}$ in constant darkness as follows: $A$, CT 15; $B$, CT $18 ; C, C T 21 ; D$, CT $24 ; E$, CT $3 ; F, C T 6 ; G$, CT $9 ; H$, CT 12 . Light pulses initiated late in the subjective day and early in the subjective night (CT $6,9,12$, and 15 ) produced delays of the circadian oscillation, while those initiated late in the subjective night (CT 18, 21, and 24 ) produced advances of the circadian oscillation. 


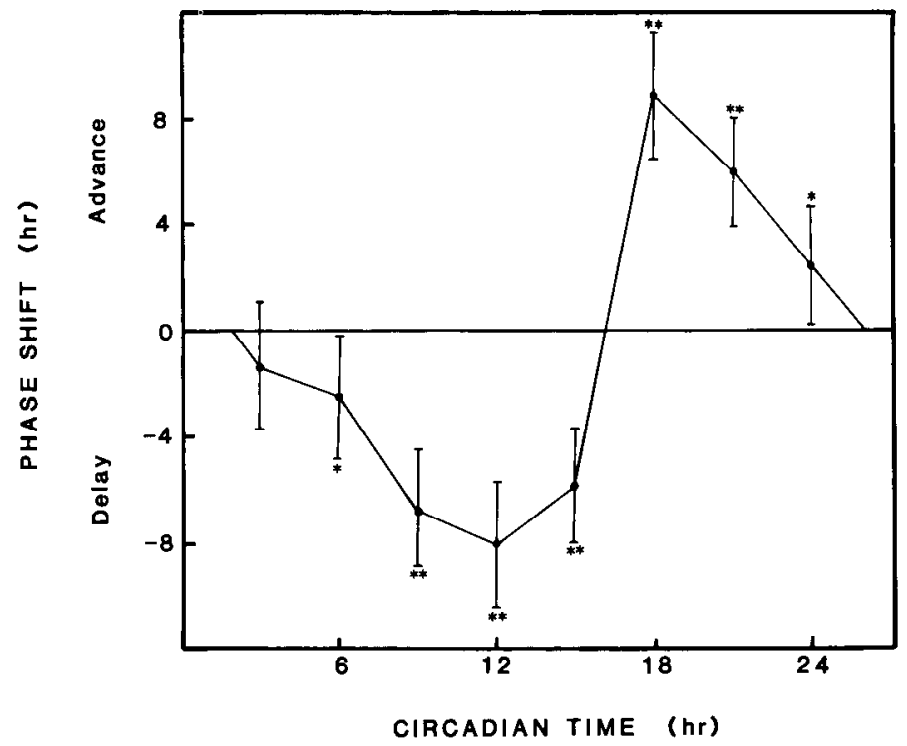

Figure 6. Phase-response curve: The dependence of the magnitude and direction of the phase-shift response upon the phase at which the light pulse was initiated. Phase for each cell chamber was analyzed during the second cycle following the light pulse, and the phase difference between chambers that received a light pulse and chambers maintained in constant darkness was calculated. The midpoint of the cycle was used as a phase reference point. Points and bars represent mean $\pm 95 \%$ confidence limits for 4-5 experimental and 4-5 control cell chambers depending on the experiment. Analysis of variance revealed significant difference among treatment means $(p<0.001, d f=37)$. Significance for each time point was determined using Student's $t$ test: ${ }^{*} p<0.05$, ${ }^{* *} p<0.001$, except for CT 9 where ${ }^{* *} p<0.002$.

CT 15 and CT 18, light exposure caused modest reductions; whereas at the other phases, melatonin release was reduced to levels similar to those seen during the day in the LD 12:12 light cycle. There is an interesting correlation between the effects of light pulses on the amplitude of the rhythm and the effects of light on melatonin release. For both parameters, the effects of light are reduced at CT 15 and CT 18. There does not appear to be any simple relationship among the light-induced effects on the magnitude of the phase shift, the increase in amplitude and the inhibition of melatonin release. However, changes in the coherence of the population of oscillators caused by light treatment could account for these changes in the waveform of the melatonin rhythm.

\section{Discussion}

Circadian oscillations are regulated by environmental light-dark cycles through the process of entrainment, which involves the control of both the phase and period of the rhythm (Pittendrigh, 1981). Although entrainment has been analyzed extensively in a number of organisms in vivo, there are few preparations from multicellular organisms in which this physiological process can be studied in vitro (Takahashi and Zatz, 1982; Jacklet, 1984). The best known examples are the isolated eyes of the marine mollusks, Aplysia californica and Bulla goldiana, which contain circadian oscillators with photoreceptive input (Eskin, 1979; Block and McMahon, 1984). Among vertebrates, the pineal of nonmammalian species (chick: Takahashi et al., 1980; Anolis: Menaker and Wisner, 1983) and the retina of amphibians (Xenopus: Besharse and Iuvone, 1983) appear to be unique in having both circadian oscillators and photoreceptors located together.

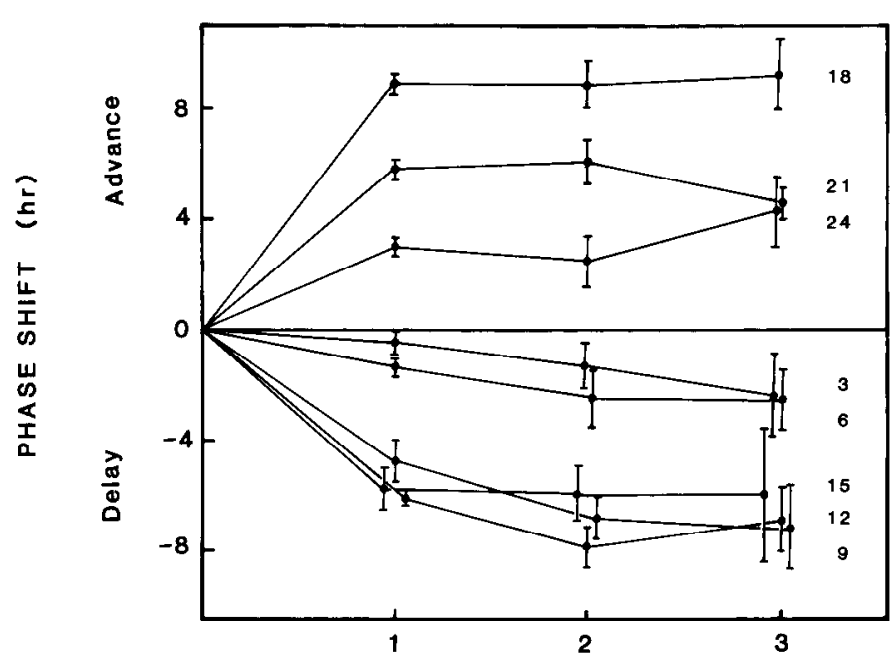

CYCLES AFTER LIGHT PULSE

Figure 7. Time course of phase shift to $6 \mathrm{hr}$ light pulses. The difference in the phase of the rhythm between chambers that received a light pulse and chambers maintained in constant darkness was calculated during the cycles following the light pulse. The midpoint of the cycle was used as a phase reference point. Points and bars represent mean \pm SEM for 4-5 experimental and 4-5 control cell chambers depending on the experiment. The light pulse was initiated at the circadian times indicated (CT 15, 18, 21, 24, 3, 6, 9, 12).

Therefore, future work directed towards a cellular understanding of the entrainment process will depend upon analysis of one of these systems in vitro. In this paper we have shown that the circadian oscillation of melatonin release expressed by dissociated pineal cell cultures can be entrained by light in vitro. Cyclic light conditions (LD 12:12) regulate the phase of the melatonin rhythm measured after release of the cultures into constant darkness, demonstrating that the steady-state phase of the underlying circadian oscillators was shifted. Furthermore, single light pulses shift the phase of the melatonin rhythm in a phase-dependent manner. Pulses in the early subjective night cause delay phase shifts, and pulses in the late subjective night cause advance phase shifts. The phase-response curve to light pulses for chick pineal cells is remarkably similar to the phaseresponse curves that have been measured for behavioral and pineal rhythms in birds (Eskin, 1971; Binkley et al., 1981).

Like the isolated pineal organ (Takahashi and Menaker, 1984b), dissociated chick pineal cell cultures can be described functionally as a system containing at least 3 major components: (1) an input pathway that is photoreceptive; (2) a circadian oscillator or pacemaker that generates the rhythm; and (3) an output pathway that results in the synthesis of melatonin. For entrainment to occur, the photoreceptor must be coupled to the oscillator; and to generate rhythmic melatonin output, the oscillator must be coupled to the melatonin synthctic pathway. We know from tissue reduction experiments and dissociated cell culture experiments that this 3 component system is redundant and does not depend upon normal tissue organization (Deguchi, 1979b; Takahashi and Menaker, 1984b; Robertson and Takahashi, 1988). The expression of these 3 components in dissociated cell cultures raises a number of questions. Are all 3 functions properties of a single cell type or, alternatively, are they distributed in more than one cell type? Does the generation of circadian rhythmicity require cellular interaction or is it a 

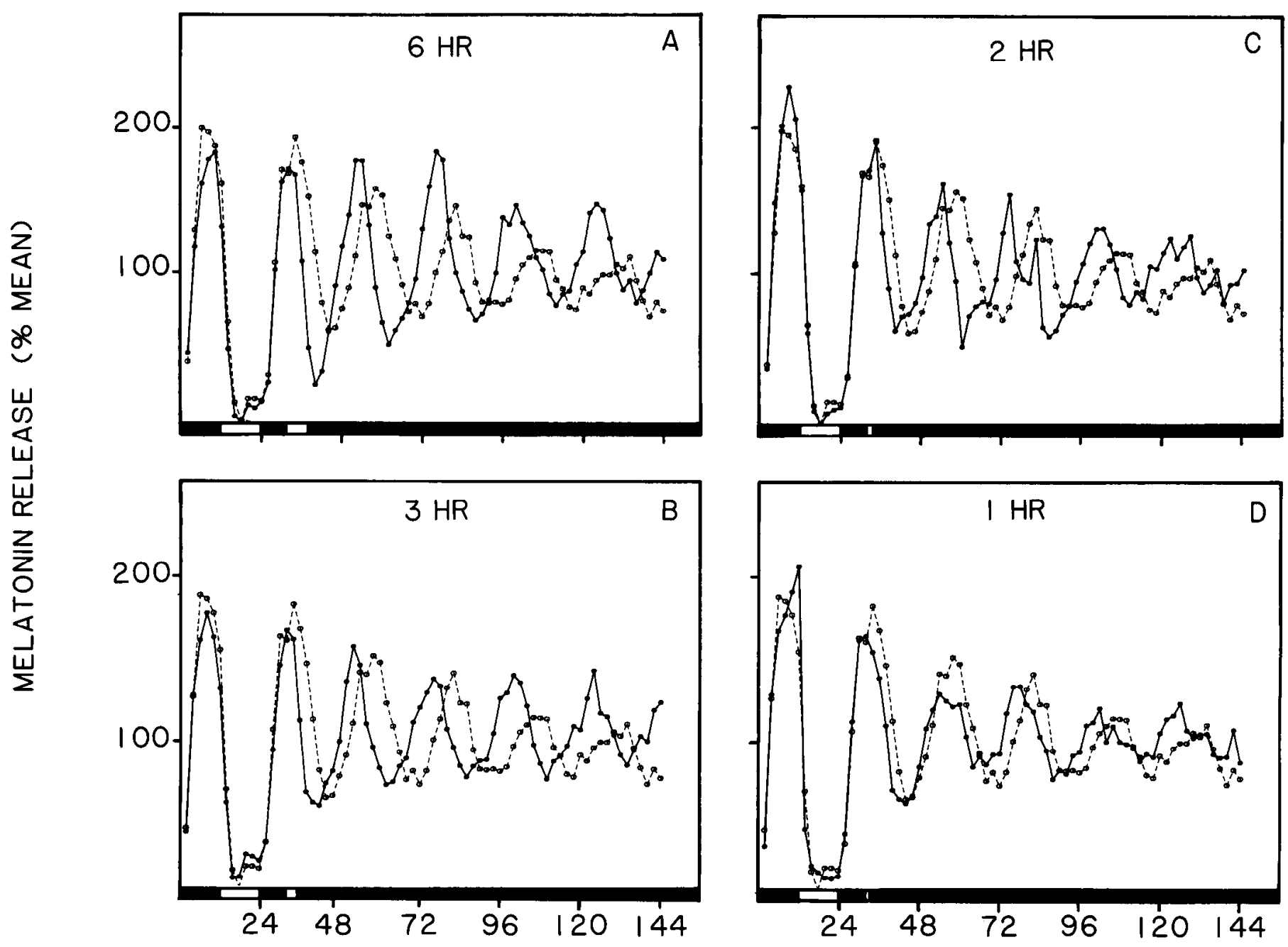

TIME IN HOURS

Figure 8. Effects of light pulse duration on the phase shift of the melatonin rhythm from dispersed chick pincal cells. Dark bars indicate the lightdark cycle. Closed circles represent the ensemble average of 4 cell chambers that received a pulse of light beginning at CT 20 . Open circles represent the ensemble average of 4 cell chambers maintained in constant darkness. The duration of the light pulses were as follows: $A, 6 \mathrm{hr} ; B, 3 \mathrm{hr} ; C, 2$ hr; $D, 1$ hr.

cellular property? As we described in the previous paper (Robertson and Takahashi, 1988), there appear to be at least 3 major cell types in the cultures: pinealocytes, interstitial cells, and fibroblastic cells. Anatomical evidence from intact avian pineal tissue suggests that the photoreceptors in the pineal gland are pinealocytes (Collin and Oksche, 1981). [Although in chicken pineal there appear to be 2 morphological subtypes of pinealocytes: those that are anatomically photoreceptorlike and those that resemble secretory cells (Bischoff, 1969).] If the pinealocytes in cell cultures from chick are photoreceptive, then at least 2 of the functions would be located in the same cell type. The key question remains, however: Are the circadian oscillators contained in the pinealocytes? At present we do not have a direct test of this hypothesis. However, the cell cultures used in these experiments employ a rather low cell density and anatomical connections among the cells on the microcarriers are restricted to the few cells on each bead. Thus, although we have not addressed this issue directly, it appears unlikely that extensive anatomical connections are required for the expression of the 3 functions. We cannot, of course, eliminate the possibility of intercellular communication through humoral routes.

Until recently, most investigators working at the cellular and molecular level have utilized microorganisms and invertebrates as model systems for studying circadian rhythms, e.g., Acetabularia, Chlamydomonas, Euglena, Gonyaulax, Neurospora, Drosophila, Aplysia, and Bulla (Hastings and Schweiger, 1975; Feldman, 1982; Jacklet, 1984). In the last decade, the localization of structures that control circadian rhythms among the vertebrates has led to the development of new model systems for cellular analysis (Takahashi and Zatz, 1982). These include the avian pineal (Takahashi et al., 1980), the Anolis pineal (Menaker and Wisner, 1983), the eye of Xenopus (Besharse and Iuvone, 1983), and the mammalian suprachiasmatic nucleus (Green and Gillette, 1982; Earnest and Sladek, 1986). The expression of circadian rhythms and photic entrainment in dissociated chick pineal cell cultures should pcrmit a mechanistic analysis of the 


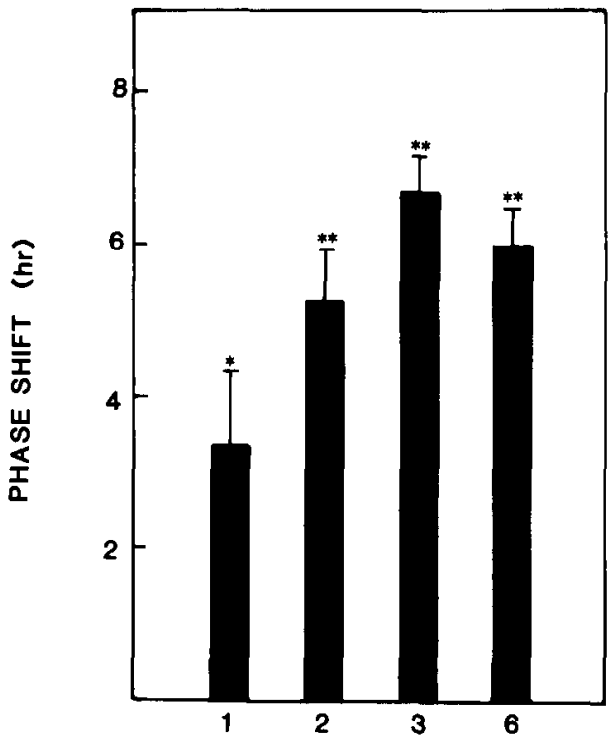

LIGHT PULSE DURATION (hr)

Figure 9. Quantitative analysis of phase shifts obtained with 1, 2, 3, and $6 \mathrm{hr}$ light pulses beginning at CT 20 . The difference in phase between 4 replicate cell chambers that received a light pulse and 4 replicate cell chambers maintained in constant darkness was determined for the second cycle following the light puise. The midpoint of the cycle was used as a phase reference point. Error bars represent SEM. Analysis of variance revealed a significant difference among treatment means $(p<$ $0.001, d f=15$ ). Significance for each duration was determined using Student's $t$ test: ${ }^{*} p<0.02,{ }^{* *} p<0.001$.

generation and entrainment of a vertebrate circadian pacemaking system. With the development of vertebrate preparations, it will be interesting to see whether similarities in mechanism exist both among vertebrate classes and among distantly related phylogenetic groups.

\section{References}

Besharse, J. C., and P. M. Iuvone (1983) Circadian clock in Xenopus eye controlling retinal serotonin $\mathrm{N}$-acetyltransferase. Nature 305: 133 135.

Binkley, S., G. Muller, and T. Hernandez (1981) Circadian rhythm in pineal $\mathrm{N}$-acetyltransferase activity: Phase shifting by light pulses (I). J. Neurochem. 37: 798-800.

Bischoff, M. B. (1969) Photoreceptoral and secretory structures in the avian pineal organ. Ultrastruct. Res. 28: 16-26.

Block, G. D., and D. G. McMahon (1984) Cellular analysis of the Bulla ocular circadian pacemaker system. III. Localization of the circadian pacemaker. J. Comp. Physiol. 155: 387-395.

Collin, J. P. and A. Oksche (1981) Structural and functional relationships in the nonmammalian pineal gland. In The Pineal Gland, Vol. 1, Anatomy and Biochemistry, R. J. Reiter, ed., pp. 27-67, CRC Press, Boca Raton, FL.
Deguchi, T. (1979a) Circadian rhythm of serotonin N-acetyltransferase activity in organ culture of chicken pineal gland. Science 203 . $1245-1247$.

Deguchi, T. (1979b) A circadian oscillator in cultured cells of chicken pineal gland. Nature 282: 94-96.

Deguchi, T. (1981) Rhodopsin-like photosensitivity of isolated chicken pineal gland. Nature 290: 706-707.

Earnest, D. J., and C. D. Sladek (1986) Circadian rhythms of vasopressin release from individual rat suprachiasmatic explants in vitro. Brain Res. 382: 129-133.

Enright, J. T. (1984) Mutual excitation of damped oscillators and selfsustainment of circadian rhythms. In Mathematical Models of the Circadian Sleep-Wake Cycle, M. C. Moore-Ede and C. A. Czeisler, eds., pp. 1-15, Raven, New York.

Eskin, A. (1971) Some properties of the system controlling the circadian activity rhythm of sparrows. In Biochronometry, M. Menaker, ed., pp. 55-78, National Academy of Science, Washington, D.C.

Eskin, A. (1979) Circadian system of the Aplysia eye: Properties of the pacemaker and mechanisms of its entrainment. Fed. Proc. 38: 2573-2579.

Feldman, J. F. (1982) Genetic approaches to circadian clocks. Annu. Rev. Plant. Physiol. 33: 583-608.

Green, D. J., and R. Gillette (1982) Circadian rhythm of firing rate recorded from single cells in the rat suprachiasmatic brain slice. Brain Res. 245: 198-200.

Hamm, H., J. S. Takahashi, and M. Menaker (1983) Light-induced decrease of serotonin $\mathrm{N}$-acetyltransferase activity and melatonin in the chicken pineal gland and retina. Brain Res. 266: 287-293.

Hastings, J. W., and H.-G. Schweiger (1975) The Molecular Basis of Circadian Rhythms, Dahlem Konferenzen, Berlin.

Jacklet, J. W. (1984) Neural organization and cellular mechanisms of circadian pacemakers. Int. Rev. Cytol. 89: 25l-294.

Kasal, C. A., and J. R. Perez-Polo (1980) In vitro evidence of photoreception in the chick pineal gland and its interaction with the circadian clock controlling $\mathrm{N}$-acetyltransferase (NAT). J. Neurosci. Res. 5: 579-585.

Menaker, M., and S. Wisner (1983) Temperature-compensated circadian clock in the pineal of Anolis. Proc. Natl. Acad. Sci. USA 80 6119-6121.

Pittendrigh, C. S. (1981) Circadian systems: Entrainment. In Handbook of Behavioral Neurobiology, Biological Rhythms, Vol. 4, J. Aschoff, ed., pp. 57-80, Plenum, New York.

Robertson, L. M., and J. S. Takahashi (1988) Circadian clock in cell culture: I. Oscillation of melatonin release from dissociated chick pineals cell in flow-through microcarrier culture. J. Neurosci. 8: 1221.

Takahashi, J. S., and M. Menaker (1984a) Circadian rhythmicity: Regulation in the time domain. In Biological Regulation and Development, Vol. 3B, K. R. Yamamoto and R. F. Goldberger, eds., pp. 285-303, Plenum, New York.

Takahashi, J. S., and M. Menaker (1984b) Multiple redundant circadian oscillators within the isolated avian pineal gland. J. Comp. Physiol. 154: 435-440.

Takahashi, J. S., and M. Zatz (1982) Regulation of circadian rhythmicity. Science 217: 1104-1111.

Takahashi, J. S., H. Hamm, and M. Menaker (1980) Circadian rhythms of melatonin release from individual superfused chicken pineal glands in vitro. Proc. Natl. Acad. Sci. USA 77: 2319-2322.

Wainwright, S. D., and L. K. Wainwright (1980) Regulation of cycle in chick pineal serotonin $\mathrm{N}$-acetyltransferase activity in vitro by light. J. Neurochem. 35: 451-457. 